SUBJECT AREAS:

ORGANIC-INORGANIC NANOSTRUCTURES

INORGANIC LEDS

Received 13 February 2014

Accepted

26 March 2014

Published

11 April 2014

Correspondence and requests for materials should be addressed to H.O. (haou@fotonik. dtu.dk) \section{Broadband Antireflection and Light} Extraction Enhancement in Fluorescent $\mathrm{SiC}$ with Nanodome Structures

\author{
Yiyu Ou', Xiaolong Zhu', Valdas Jokubavicius' ${ }^{2}$ Rositza Yakimova ${ }^{2}$, N. Asger Mortensen', \\ Mikael Syväjärvi' ${ }^{2}$ Sanshui Xiao' \& Haiyan Ou'
}

'DTU Fotonik, Technical University of Denmark, DK-2800 Lyngby, Denmark, ²Department of Physics, Chemistry and Biology, Linköping University, SE-58183 Linköping, Sweden.

We demonstrate a time-efficient and low-cost approach to fabricate $\mathrm{Si}_{3} \mathrm{~N}_{4}$ coated nanodome structures in fluorescent $\mathrm{SiC}$. Nanosphere lithography is used as the nanopatterning method and $\mathrm{SiC}$ nanodome structures with $\mathrm{Si}_{3} \mathrm{~N}_{4}$ coating are formed via dry etching and thin film deposition process. By using this method, a significant broadband surface antireflection and a considerable omnidirectional luminescence enhancement are obtained. The experimental observations are then supported by numerical simulations. It is believed that our fabrication method will be well suitable for large-scale production in the future.

T he high-quality donor-acceptor co-doped fluorescent $\mathrm{SiC}$ grown by fast sublimation growth process (FSGP) has been demonstrated to be a highly efficient wavelength-conversion material for white light-emitting diodes $(\mathrm{LEDs})^{1,2}$. Compared with the normal wavelength-conversion material like phosphors, fluorescent $\mathrm{SiC}$ is much superior in terms of high color rendering index and long lifetime. Also, $\mathrm{SiC}$ has an excellent thermal conductivity and a small lattice mismatch with GaN (critical material for blue LEDs), thus making itself a wellestablished substrate material for nitride growth. However, $\mathrm{SiC}$ has a rather high refractive index (2.65 at $580 \mathrm{~nm})^{3}$, indicating that most of the emitted light is confined in the material and can not escape into air due to the total internal reflection, i.e. a very low extraction efficiency. To enhance the light extraction efficiency of the SiC-based white LED, different nanopatterning approaches (e-beam lithography, self-assembled metal nanoparticles etc.) have been developed to fabricate an antireflection structure (ARS) array on a $\mathrm{SiC}$ surface ${ }^{4-6}$. Among these methods, a metal template is always needed to resist a strong etching power in order to break the strong atomic binding energy of the $\mathrm{SiC}$. However, metals are routinely excluded by a lot of semiconductor processing facilities and they are rather expensive when considering any future mass production. Therefore we propose a metal-free method, i.e, using self-assembled polystyrene (PS) nanospheres as a template, to fabricate nanodome structures on nitrogen-boron co-doped fluorescent $6 \mathrm{H}-\mathrm{SiC}$ by using reactive-ion etching (RIE) process. Compared to the commonly used e-beam lithography and nanoimprint lithography processes, nanosphere lithography is a time-saving and cost-efficient nanopatterning technique for subsequent etching approaches and it is also a promising way for large-scale production ${ }^{7-13}$. Moreover, self-assembly nanospheres with different material and dimension choices are commercially available, thus providing an attractive platform in various applications.

In the paper, $\mathrm{B}$ and $\mathrm{N}$ co-doped fluorescent $6 \mathrm{H}$-SiC samples ( $200 \mu \mathrm{m}$ grown by FSGP) were processed by nanosphere lithography and reactive ion etching (RIE). The wavelength-scale nanodome structures formed at the surface suppress the average surface reflection over the whole visible spectral range and enhance the omnidirectional luminescence. The antireflection and luminescence enhancement performance of the nanodome structures are further enhanced by depositing a thin $\mathrm{Si}_{3} \mathrm{~N}_{4}$ coating on top of the nanodome structures. To the best of our knowledge, this is the first report using PS nanospheres as template to form nanodome structures on SiC.

\section{Results}

A schematic drawing of the detailed fabrication process of the nanodome structures is illustrated in Fig. 1. Firstly, a monolayer hexagonal-close-packed array of PS nanospheres with a diameter of $600 \mathrm{~nm}$ (size dispersion of 1\%) was formed on a pre-treated SiC sample surface by a self-assembly method (see Fig. 1(a) $)^{14-16}$. Subsequently, the $\mathrm{SiC}$ sample was subjected to RIE (STS cluster system C010) for pattern transfer, where the PS nanospheres 
(a)

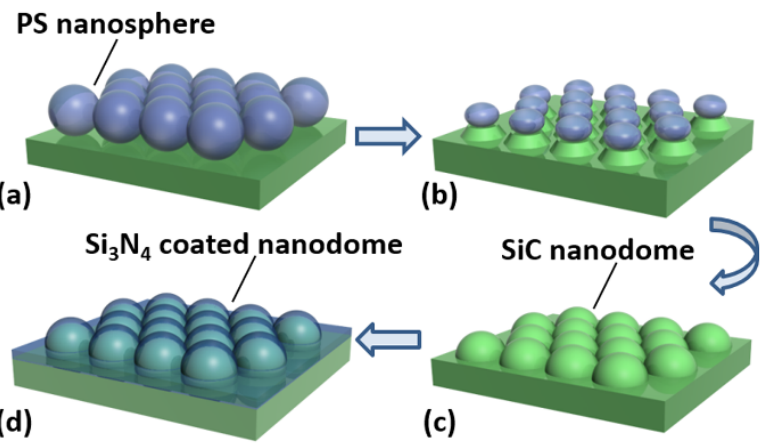

Figure $1 \mid$ Schematic diagram showing the detailed fabrication process of $\mathrm{Si}_{3} \mathrm{~N}_{4}$ coated nanodome structures on fluorescent $\mathrm{SiC}$ samples.

(a) Formation of self-assembled polystyrene monolayer nanospheres as etching template, (b) dry etching process by RIE with $\mathrm{SF}_{6}$ and $\mathrm{O}_{2}$ gases, (c) formation of the nanodome structures on fluorescent $\mathrm{SiC}$ samples and, (d) $\mathrm{Si}_{3} \mathrm{~N}_{4}$ film deposition on $\mathrm{SiC}$ nanodome by PECVD.

monolayer serves as an etching template (see Fig. 1(b)). Due to the isotropic nature of the chemical etching from the plasma gases and directional bombardment of accelerated ions from the physical etching in RIE, the PS nanospheres were etched away in an anisotropic way. Consequently, the nanospheres became thinner and their transverse diameters also decreased gradually. The exposed area of the underlying $\mathrm{SiC}$ substrate then increased. After 5 minutes etching, the nanospheres are consumed completely and $\mathrm{SiC}$ nanodome structures are achieved by using the PS nanospheres (shrinking during the etching) as the unique template (see Fig. 1(c)). In addition, a $57 \mathrm{~nm}$ thick $\mathrm{Si}_{3} \mathrm{~N}_{4}$ coating with an intermediate refractive index ( $\mathrm{n}$ $=2.0)$ between the value of air $(\mathrm{n}=1)$ and $6 \mathrm{H}-\mathrm{SiC}(\mathrm{n}=2.65)$ was deposited on top of the nanodome structures by plasma-enhanced chemical vapor deposition (PECVD) to further enhance the extraction efficiency.

Oblique-view SEM images of the close-packed monolayer PS nanospheres, $\mathrm{SiC}$ nanodome structures $(\mathrm{ND}-\mathrm{SiC})$, and $\mathrm{Si}_{3} \mathrm{~N}_{4}$ coated nanodome structures (CND-SiC) are demonstrated in Figs. 2(a)2(c), respectively. A large view SEM image is inset in Fig. 2(a) to show the scalability of this method. The nanodome structure has a structure height of around $200 \mathrm{~nm}$ (measured directly from crosssection SEM image) with a semi-spherical shape and smooth surface. Meanwhile, the aspect ratio of the nanodome structure is low which is a desired property for further $\mathrm{Si}_{3} \mathrm{~N}_{4}$ film deposition. On the other hand, in Fig. 2(c), the $\mathrm{Si}_{3} \mathrm{~N}_{4}$ coated nanodome exhibits a larger structure height and a relatively rough surface. Fig. 2(d) shows a photograph of the $\mathrm{SiC}$ sample with a partially plain surface (plain-SiC) and a partially coated nanodome covered surface(coated nanodome). The surface color of the sample turns from bright grey to black after surface patterning, indicating a significant suppression of surface reflection.

The measured reflectance spectra in a wavelength range of 390$785 \mathrm{~nm}$ are shown in Fig. 3(a). In the measured spectral range, the average reflectance of plain- $\mathrm{SiC}$ is around $20.5 \%$. Due to the graded refractive index profile of ND-SiC, the average reflectance is significantly suppressed to $2.0 \%$, while the reflectance is below $4 \%$ throughout the whole wavelength range. A lower average reflectance of $0.99 \%$ is obtained for the CND-SiC sample. This is because the $\mathrm{Si}_{3} \mathrm{~N}_{4}$ coating further relieves the refractive index difference between the air/SiC interfaces. The above experimental observations are explained by the effective refractive index $\left(n_{E}\right)$ profiles of the interfaces between air and different SiC samples, as shown in Figs. $3(\mathrm{~b})$ to $3(\mathrm{~d})$. The $\mathrm{n}_{\mathrm{E}}$ profile could be approximated by $n_{E}=f n_{1}+(1-f) n_{2}{ }^{17}$, where $n_{1}$ and $\mathrm{n}_{2}$ are the refractive indices of fluorescent $\mathrm{SiC}(\mathrm{n}=2.65)$ and air $(\mathrm{n}=1)$ respectively, and $\mathrm{f}$ is the filling factor weighted by the structure volume at different structure heights. As shown in Fig. 3(b), the
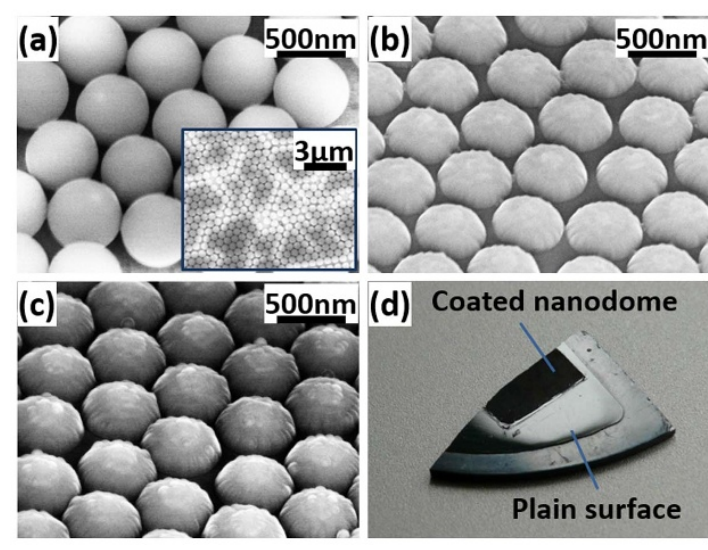

Figure 2 $\mid$ Oblique-view SEM images of the (a) monolayer hexagonalclose-packed polystyrene nanospheres (inset is a large-scale view), (b) fabricated SiC nanodome structures, and (c) Si3N4 coated nanodome structures respectively. (d) A photograph of SiC sample with a partially plain surface (lower right part) and partially covered by the coated nanodome structures (upper left part).

refractive index changes abruptly from 2.65 to 1 across the plain interface, which causes large internal/surface reflection. For the $\mathrm{ND}-\mathrm{SiC}$, the diameter of the dome structure shrinks gradually from the bottom to the top, thus resulting in a graded transition of the $n_{E}$ (see Fig. 3(c)). As a result, nanodome structures are anticipated to achieve considerable reflection suppression over a broadband spectral range. Compared with $\mathrm{ND}-\mathrm{SiC}, \mathrm{CND}-\mathrm{SiC}$ with a $\mathrm{Si}_{3} \mathrm{~N}_{4}$ coating which has an intermediate refractive index $(\mathrm{n}=2.0)$ can further relieve the refractive index difference in a more smooth way (see Fig. 3(d)), thus an even lower surface reflection could be expected. These expectations are supported by our experimental results shown in Fig. 3(a). The choice of $\mathrm{Si}_{3} \mathrm{~N}_{4}$ coating is just for the proof-ofconcept. Further suppression of the antireflection could be achieved by carefully engineering the refractive index and thickness of the coating.

In order to estimate the influence of the sphere diameter on the optical antireflection enhancement, we illustrate in Fig. 4 the total optical reflectance of ND-SiC and CND-SiC as a function of the sphere diameter calculated by a rigorous coupled-wave analysis (RCWA) model. The sphere diameter varies between 300 and $900 \mathrm{~nm}$. In general, CND-SiC has even lower reflectance than ND$\mathrm{SiC}$. We again observe a fairly low reflectance for both ND-SiC and $\mathrm{CND}-\mathrm{SiC}$ with a diameter of $600 \mathrm{~nm}$ as we used (the dash line in the figure), especially in the wavelength region of 550-650 $\mathrm{nm}$ where the luminescence peak of $\mathrm{SiC}$ is located. As seen from Fig. 5(a), the fluorescent $\mathrm{SiC}$ emits at peak wavelength of $575 \mathrm{~nm}$, therefore $600 \mathrm{~nm}$ diameter nanospheres were chosen as the template with the guidance of the simulation results in Fig. 4. Moreover, by tuning the sphere size, the spectral response to the antireflection can be adjusted accordingly. Therefore, such feature can be applied for different wavelength purpose.

For the two orthogonal polarizations, both the luminescence spectrum at the emission angle normal to the sample surface and the angle-resolved photoluminescence are quite similar to each other. So results for only one polarization are shown in Fig. 5. Fig. 5(a) shows that the fluorescent $\mathrm{SiC}$ sample exhibits a broad luminescence peaked at $575 \mathrm{~nm}$ and the luminescence is enhanced after surface nanostructuring. Compared to plain-SiC, the ND-SiC demonstrates a $107 \%$ luminescence enhancement at the emission angle normal to the sample surface. Meanwhile, the luminescence of the CND-SiC is enhanced significantly by $138 \%$. The observed photoluminescence enhancement results are in a good consistency with the experimental observations from the surface reflectance suppression measurements. 
(a)

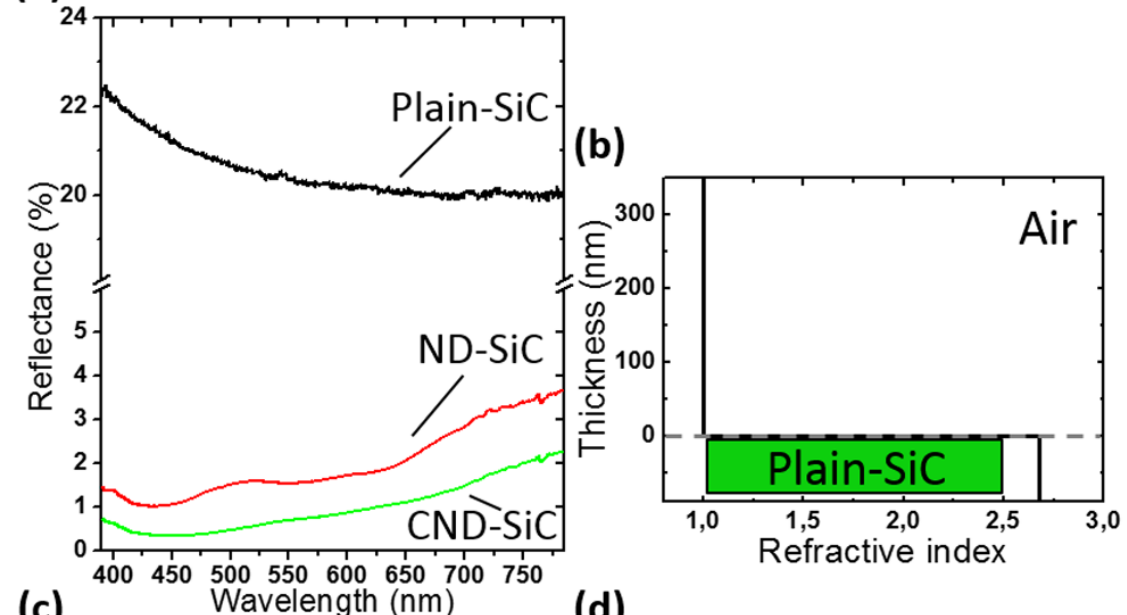

(c)

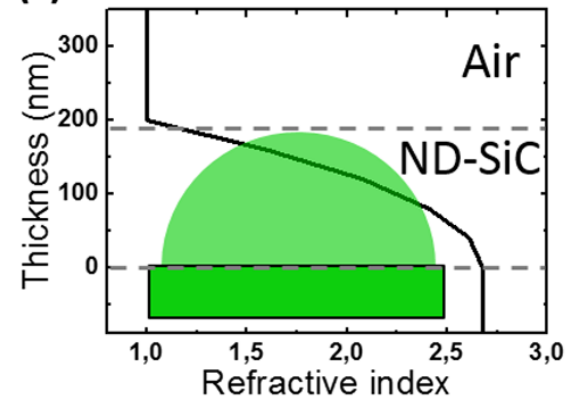

(d)

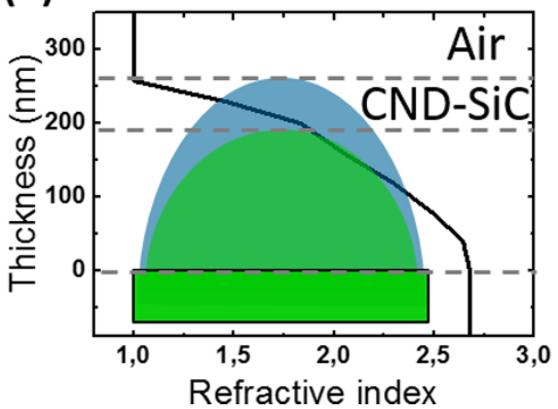

Figure $3 \mid$ (a) Measured optical reflectance of plain-SiC, ND-SiC, and CND-SiC. (b-d) Schematic illustrations and calculated effective refractive index profiles of plain-SiC, ND-SiC, and CND-SiC respectively.

Angular integral emission intensity profiles from angle-resolved photoluminescence measurements are also illustrated in Fig. 5(b) for plain-SiC, ND-SiC, and CND-SiC samples. A broadband extraction enhancement is obtained for both ND-SiC and CND-SiC samples at all the tested angles. Especially, a large enhancement over $50 \%$ is obtained at a large emission angle up to $60^{\circ}$ for both samples.

\section{Discussion}

Compared to periodic nanocone structures in Ref. 5, nanodome structures in this paper has very close luminescence enhancement effect. The merit of these nanodome structures is they have much lower aspect ratio than nanocone structures, thus it could benefit from a capping layer with medium refractive index (like $\left.\mathrm{Si}_{3} \mathrm{~N}_{4}\right)$ to further enhance the extraction efficiency.

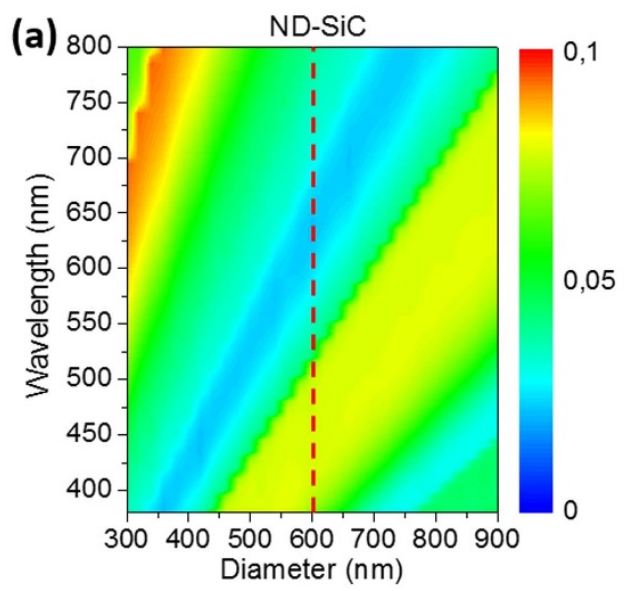

In conclusion, we have demonstrated a novel approach to fabricate nanodome structures in fluorescent $\mathrm{SiC}$. This attractive approach relies on a RIE process with self-assembled monolayer PS nanospheres as a template and a thin film deposition process. Compared with a plain sample, the coated nanodome structures present a graded transition of the effective refractive index from the structure bottom to the surface which leads to a significant reflectance reduction from $20.5 \%$ to $0.99 \%$ in the spectral range of $390-785 \mathrm{~nm}$. Numerical simulations were implemented to further support the experimental results. A considerable luminescence enhancement of $138 \%$ is also achieved and large luminescence enhancement can be maintained in a large emission angle range. Related applications such as solar cells and optical detectors could also benefit from the excellent antireflection

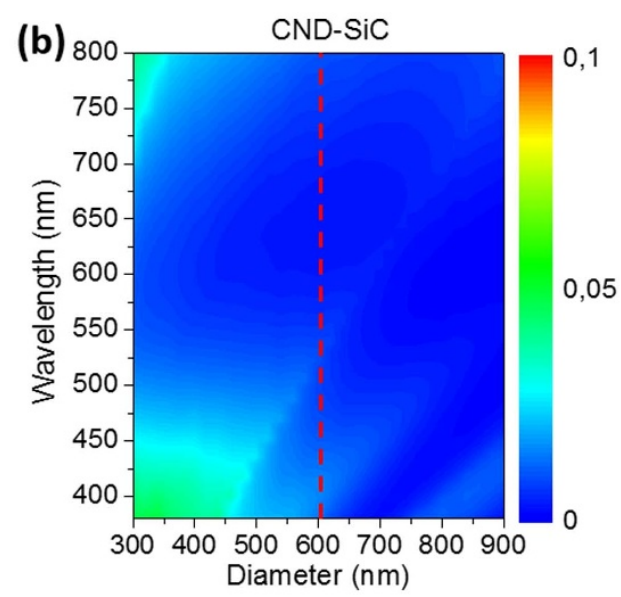

Figure 4 Simulated reflectance of the SiC sample as a function of wavelength and nanosphere diameter for (a) ND-SiC and (b) CND-SiC. 

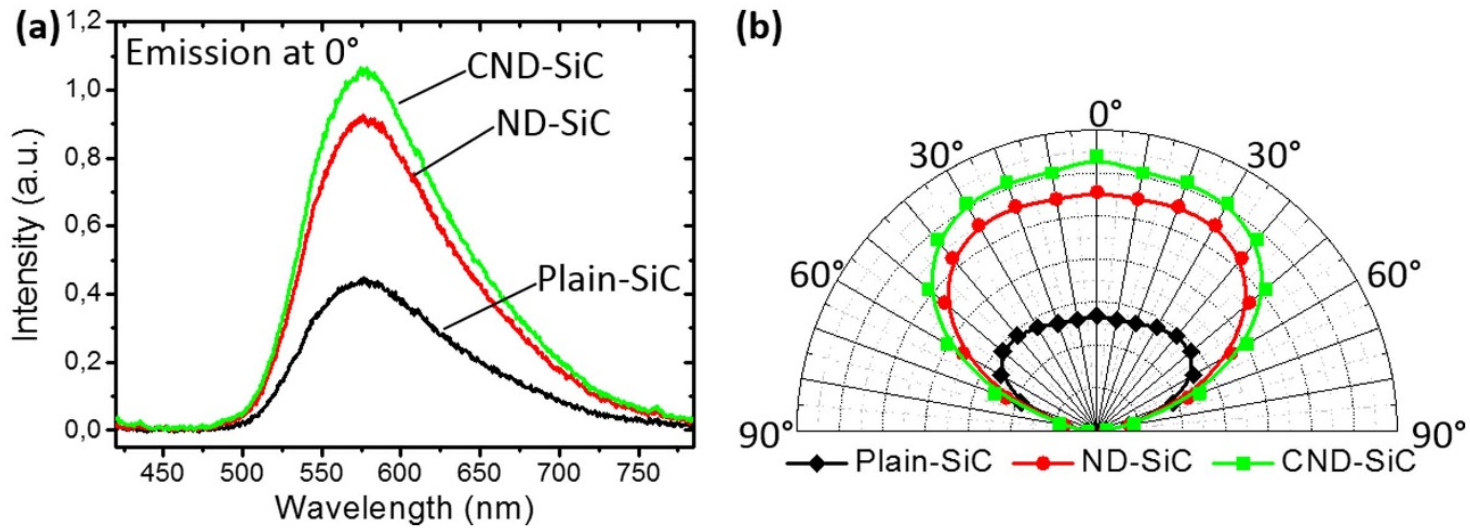

Figure $5 \mid$ (a) Photoluminescence spectra at emission angle of $0^{\circ}$ and (b) angular emission profiles of plain-SiC, ND-SiC, and CND-SiC respectively.

performance by using this low-cost and scalable fabrication method.

\section{Methods}

Optimized RIE conditions were applied with a R.F. power of $100 \mathrm{~W}$, chamber pressure of $30 \mathrm{mT}$, and a mixture of $\mathrm{SF}_{6}$ and $\mathrm{O}_{2}$ gases $\left(24\right.$ and $6 \mathrm{sccm}$ ). The $\mathrm{Si}_{3} \mathrm{~N}_{4}$ thin film was deposited by PECVD (STS cluster system C010). The thickness of $\mathrm{Si}_{3} \mathrm{~N}_{4}$ thin film is measured on a dummy wafer, which has a plain surface and is deposited at the same time as the nanodome sample, by using filmtek (Filmtek 4000 Spectrometer). The etched profile and surface morphology of the processed SiC samples were then observed by a scanning electron microscope (SEM LEO 1550, Carl Zeiss). Surface reflectance measurements of different $\mathrm{SiC}$ samples were carried out by using a goniometer system (Instrument Systems, GON360). A halogen lamp as a broadband light source was connected to the transmitter arm of the goniometer and the receiver arm was connected to an optical spectrometer (Instrument Systems, CAS140B). SiC samples were mounted on the sample stage and the reflection spectra were measured with an incidence angle of $8^{\circ}$ with respect to the surface-normal direction. Angleresolved photoluminescence measurements were carried out by using the same goniometer. A $377 \mathrm{~nm}$ diode laser was connected to the transmitter arm as the excitation light source and the $\mathrm{SiC}$ sample was optically excited from its back side at room temperature. The detection angle of the receiver arm was varied from 0 (surface-normal direction) to $85^{\circ}$ with a step of $10^{\circ}$ and the corresponding photoluminescence spectra at two orthogonal polarizations were then acquired.

1. Ou, Y. et al. Donor-acceptor-pair emission characterization in N-B doped fluorescent SiC. Opt. Mater. Express 1, 1439-1446 (2011).

2. Kamiyama, S. et al. Extremely high quantum efficiency of donor-acceptor-pair emission in N-and-B-doped 6H-SiC. J. Appl. Phys. 99, 093108 (2006).

3. Shaffer, P. T. B. Refractive index, dispersion, and birefringence of silicon carbide polytypes. App. Optics 10, 1034-1936 (1971).

4. Argyraki, A., Ou, Y. \& Ou, H. Broadband antireflective silicon carbide surface produced by cost-effective method. Opt. Mater. Express 3, 1119-1126 (2013).

5. Ou, Y. et al. Broadband and omnidirectional light harvesting enhancement of fluorescent SiC. Opt. Express 20, 7575-7579 (2012).

6. Kawai, R. et al. Realization of extreme light extraction efficiency for moth-eye LEDs on SiC substrate using high-reflection electrode. Phys. Status Solidi C 7, 2180-2182 (2010).

7. Park, H. et al. Broadband optical antireflection enhancement by integrating antireflective nanoislands with silicon nanoconical-frustum arrays. Adv. Mater 23, 5796-5800 (2011).

8. Sanatinia, R. et al. GaAs nanopillar arrays with suppressed broadband reflectance and high optical quality for photovoltaic applications. Opt. Mater. Express 2, 1671-1679 (2012).

9. Zhu, J., Hsu, C.-M., Yu, Z., Fan, S. \& Cui, Y. Nanodome solar cells with efficient light management and self-cleaning. Nano Lett. 10, 1979-1984 (2010).

10. Phillips, B. M., Jiang, P. \& Jiang, B. Biomimetic broadband antireflection gratings on solar-grade multicrystalline silicon wafers. Appl. Phys. Lett. 99, 191103 (2011).
11. Wei, T. et al. Selectively grown photonic crystal structures for high efficiency InGaN emitting diodes using nanospherical-lens lithography. Appl. Phys. Lett. 101, 211111 (2012).

12. Fu, W. Y., Wong, K. K.-Y. \& Choi, H. W. Close-packed hemiellipsoid arrays: A photonic band gap structure patterned by nanosphere lithography. Appl. Phys. Lett. 95, 133125 (2009)

13. Chang, Y.-C., Wang, S.-M., Chung, H.-C., Tseng, C.-B. \& Chang, S.-H. Observation of absorption-dominated bonding dark plasmon mode from metalinsulator-metal nanodisk arrays fabricated by nanospherical-lens lithography. ACS Nano 6, 3390-3396 (2012).

14. Zhu, X. et al. Broadband enhancement of spontaneous emission in a photonicplasmonic structure. Opt. Lett. 37, 2037-2039 (2012).

15. Ou, Y., Zhu, X., Møller, U., Xiao, S. \& Ou, H. Broadband antireflection nanodome structures on $\mathrm{SiC}$ substrate. Paper presented at 10th IEEE Int. Conf. Group IV Photonics, Seoul, South Korea. (DOI 10.1109/Group4.2013.6644482) (2013, Aug. 28-30).

16. Zhu, X. et al. Broadband light-extraction enhanced by arrays of whispering gallery resonators. Appl. Phys. Lett. 101, 241108 (2012).

17. Motamedi, M. E., Southwell, W. H. \& Gunning, W. J. Antireflection surfaces in silicon using binary optics technology. Appl. Opt. 31, 4371-4376 (1992).

\section{Acknowledgments}

This research was supported by the Danish councils for strategic research funding (no. 09-072118), Swedish energy agency, Nordic energy research, and Swedish research council (no. 2009-5307). S. X. and X. Z. also acknowledge the support from the Catalysis for Sustainable Energy Initiative Center, funded by the Danish Ministry of Science, Technology and Innovation.

\section{Author contributions}

Y.O. and H.O. wrote the main manuscript text. Y.O. prepared figures $1-3,5$ and X.Z. prepared Fig.4. V.J. grew the f-SiC samples. X.Z. deposited nanosphere on top of f-SiC, and Y.O. did the sample processing and characterization. R.Y., M.S., S.X., N.M. and H.O. supervised the study. All authors reviewed the manuscript.

\section{Additional information}

Competing financial interests: The authors declare no competing financial interests.

How to cite this article: Ou, Y.Y. et al. Broadband Antireflection and Light Extraction Enhancement in Fluorescent SiC with Nanodome Structures. Sci. Rep. 4, 4662; DOI:10.1038/srep04662 (2014)

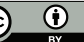

This work is licensed under a Creative Commons Attribution 3.0 Unported License. The images in this article are included in the article's Creative Commons license, unless indicated otherwise in the image credit; if the image is not included under the Creative Commons license, users will need to obtain permission from the license holder in order to reproduce the image. To view a copy of this license, visit http://creativecommons.org/licenses/by/3.0/ 